\title{
New Indications \& Dosage Forms for Existing Drugs
}

Pradaxa (dabigatranetexilate) Capsules and Oral Pellets

Patient Population Altered: June 21, 2021

Date of Original Approval: October 19, 2010

Pradaxa (dabigatranetexilate) is a direct thrombin inhibitor.

Pradaxa capsules are indicated:

- to reduce the risk of stroke and systemic embolism in adult patients with non-valvular atrial fibrillation

- for the treatment of deep venous thrombosis (DVT) and pulmonary embolism (PE) in adult patients who have been treated with a parenteral anticoagulant for 5-10 days

- to reduce the risk of recurrence of DVT and PE in adult patients who have been previously treated

- for the prophylaxis of DVT and PE in adult patients who have undergone hip replacement surgery

- for the treatment of venous thromboembolic events (VTE) in pediatric patients 8 to less than 18 years of age who have been treated with a parenteral anticoagulant for at least 5 days

- to reduce the risk of recurrence of VTE in pediatric patients 8 to less than 18 years of age who have been previously treated.

Pradaxa oral pellets are indicated:

- for the treatment of venous thromboembolic events (VTE) in pediatric patients aged 3 months to less than 12 years of age who have been treated with a parenteral anticoagulant for at least 5 days

- to reduce the risk of recurrence of VTE in pediatric patients aged 3 months to less than 12 years of age who have been previously treated.

Ayvakit (avapritinib) Tablets

New Indication Approved: June 16, 2021

Date of Original Approval: January 9, 2020
Ayvakit (avapritinib) is a kinase inhibitor for the treatment of PDGFR $\alpha$ exon 18 mutant gastrointestinal stromal tumors (GIST), and advanced systemic mastocytosis (AdvSM).

Epclusa (sofosbuvir and velpatasvir) Tablets and Oral Pellets

New Dosage Form Approved: June 10, 2021

Date of Original Approval: June 28, 2016

Epclusa (sofosbuvir and velpatasvir) is a nucleotide analog polymerase inhibitor and pan-genotypic NS5A inhibitor fixed-dose combination for the treatment of chronic genotype 1-6 hepatitis $C$ virus (HCV) infection.

Trikafta (elexacaftor/tezacaftor/ivacaftor and ivacaftor) Tablets

Patient Population Altered: June 8, 2021

Date of Original Approval: October 21, 2019

Trikafta (elexacaftor/tezacaftor/ivacaftor and ivacaftor) is a triple combination regimen for the treatment of cystic fibrosis (CF) in patients ages 6 years and older who have at least one copy of the F508del mutation in the cystic fibrosis transmembrane conductance regulator $(C F T R)$ gene or a mutation in the CFTR gene that is responsive to Trikafta based on in vitro data.

\section{Ultomiris (ravulizumab-cwvz) Injection}

Patient Population Altered: June 7, 2021

Date of Original Approval: December 21, 2018

Ultomiris (ravulizumab-cwvz) is a long-acting C5 complement inhibitor for:

- the treatment of adult and pediatric patients one month of age and older with paroxysmal nocturnal hemoglobinuria (PNH)

- the treatment of adult and pediatric patients one month of age and older with atypical hemolytic uremic syndrome (aHUS) to inhibit complementmediated thrombotic microangiopathy (TMA).

\section{Cosentyx (secukinumab) Injection}

Patient Population Altered: May 28, 2021

Date of Original Approval: January 21, 2015 
Cosentyx (secukinumab) is a selective interleukin17A (IL-17A) inhibitor for the treatment of plaque psoriasis, ankylosing spondylitis, psoriatic arthritis, and non-radiographic axial spondyloarthritis.

\section{Nurtec ODT (rimegepant) Orally Disintegrating Tablets (ODT)}

New Indication Approved: May 27, 2021

Date of Original Approval: February 27, 2020

Nurtec ODT (rimegepant) is an orally-dosed calcitonin gene-related peptide (CGRP) receptor antagonist for the acute treatment of migraine with or without aura, and the preventive treatment of episodic migraine, in adults.

\section{Zeposia (ozanimod) Capsules}

New Indication Approved: May 27, 2021

Date of Original Approval: March 25, 2020

Zeposia (ozanimod) is a sphingosine 1-phosphate receptor modulator indicated for the treatment of relapsing forms of multiple sclerosis (MS), to include clinically isolated syndrome, relapsing-remitting disease, and active secondary progressive disease in adults; and moderately to severely active ulcerative colitis (UC) in adults.

\section{Nuzyra (omadacycline) Tablets and Lyophilized Powder for Injection}

New Dosage Regimen: May 27, 2021

Date of Original Approval: October 2, 2018

Nuzyra (omadacycline) is an aminomethylcycline tetracycline antibiotic for the treatment of community-acquired bacterial pneumonia (CABP) and acute bacterial skin and skin structure infections (ABSSSI).

\section{Opdivo (nivolumab) Injection}

New Indication Approved: May 20, 2021

Date of Original Approval: December 22, 2014

Opdivo (nivolumab) is a programmed death receptor1 (PD-1) blocking antibody for the treatment of melanoma, non-small cell lung cancer, malignant pleural mesothelioma, renal cell carcinoma, classical Hodgkin lymphoma, squamous cell carcinoma of the head and neck, urothelial carcinoma, MSI-H or dMMR metastatic colorectal cancer, hepatocellular carcinoma, esophageal cancer, gastric cancer, and gastroesophageal junction cancer.

KedRAB (rabies immunoglobulin human) Injection

Patient Population Altered: May 17, 2021

Date of Original Approval: August 23, 2017

KedRAB [rabies immunoglobulin (human)] is a human plasma derived anti-rabies immunoglobulin indicated for post-exposure prophylaxis (PEP) of rabies infection.

\section{Keytruda (pembrolizumab) for Injection}

New Indication Approved: May 5, 2021

Date of Original Approval: September 4, 2014

Keytruda (pembrolizumab) is a human PD-1 (programmed death receptor-1)-blocking antibody indicated for the treatment of melanoma, non-small cell lung cancer, head and neck squamous cell carcinoma, classical Hodgkin lymphoma, primary mediastinal large B-cell lymphoma, urothelial carcinoma, microsatellite instability-high cancer, gastric cancer, esophageal cancer, cervical cancer, hepatocellular carcinoma, Merkel cell carcinoma, renal cell carcinoma, endometrial carcinoma, tumor mutational burden-high (TMB-H) cancer, cutaneous squamous cell carcinoma, and triple-negative breast cancer.

\section{Ferriprox (deferiprone) Tablets and Oral Solution}

New Indication Approved: May 1, 2021

Date of Original Approval: October 14, 2011

Ferriprox (deferiprone) is an iron chelator indicated for the treatment of transfusional iron overload in:

- thalassemia syndromes

- sickle cell disease or other anemias.

Ferriprox Tablets are indicated in adult and pediatric patients $\geq 8$ years of age and Ferriprox Oral Solution is indicated in patients $\geq 3$ years of age.

www.drugs.com/newdrugs

\section{Information collected and compiled by:}

Md. Akbar Hossain

Department of Pharmacy

ASA University of Science and Technology Bangladesh, Dhaka, Bangladesh 